\title{
Structural differences in the diaphragm of patients following controlled vs assisted and spontaneous mechanical ventilation
}

Short Running Title: VIDD in humans with spontaneous mechanical ventilation

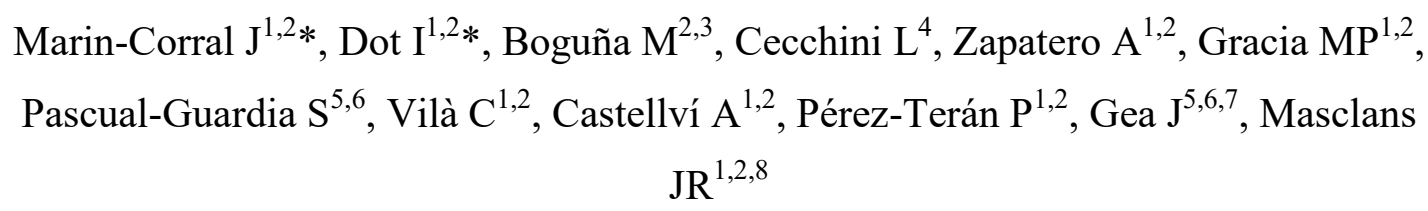

* The first two authors share the first position since they contributed equally to the study.

1 Critical Care Department, Hospital del Mar, Barcelona, Spain

2 Research Group in Critical Disorders (GREPAC), Institut Hospital del Mar d'Investigacions Mèdiques (IMIM), Barcelona, Spain

3 Universitat de Barcelona, Barcelona, Spain

4 Urology Department, Hospital del Mar, Spain

5 Respiratory Department, Hospital del Mar, IMIM, Barcelona, Spain

6 CIBER de Enfermedades Respiratorias (CIBERES)

7 DCEXS Universitat Pompeu Fabra, Barcelona, Spain

8 Medicine Department, Universitat Autònoma de Barcelona, Barcelona, Spain

Corresponding Author: Judith Marin Corral, MD, PhD, Critical Care Department, Hospital del Mar, Passeig Marítim 25-29, E-08003 Barcelona, Spain

Tel.: +34-93-2483014; fax: +34-93-2483014; e-mail: jmarincorral@gmail.com

Presented at the $30^{\text {th }}$ ESICM Lives (European Society of Intensive Care Medicine) Vienna, October 2017 as an oral presentation and at the CHEST Annual Meeting 2018, San Antonio, October 2018. Accepted as an oral presentation at the $31^{\text {st }}$ ESICM Paris, October 2018.

Competing interests: None to be declared 


\begin{abstract}
Purpose: Ventilator-induced diaphragm dysfunction or damage (VIDD) is highly prevalent in patients under mechanical ventilation (MV), but its analysis is limited by the difficulty of obtaining histological samples. In this study we compared diaphragm histological characteristics in Maastricht III (MSIII) and brain-dead (BD) organ donors and in control subjects undergoing thoracic surgery (CTL) after a period of either controlled or spontaneous MV (CMV or SMV).
\end{abstract}

Methods: In this prospective study, biopsies were obtained from diaphragm and quadriceps. Demographic variables, comorbidities, severity on admission, treatment and ventilatory variables were evaluated. Immunohistochemical analysis (fiber size and type percentages) and quantification of abnormal fibers (a surrogate of muscle damage) were performed.

Results: Muscle samples were obtained from 35 patients. MSIII ( $\mathrm{n}=16)$ had more hours on MV (either CMV or SMV) than BD ( $\mathrm{n}=14)$ and also spent more hours, and a greater percentage of time with diaphragm stimuli (time in assisted and spontaneous modalities). Cross sectional area (CSA) was significantly reduced in the diaphragm and quadriceps in both groups in comparison with CTL ( $n=5)$. Quadriceps CSA was significantly decreased in MSIII compared to BD but there were no differences in the diaphragm CSA between the two groups. Those MSIII who spent 100 or more hours without diaphragm stimuli presented reduced diaphragm CSA without changes in their quadriceps CSA. Proportion of internal nuclei in diaphragms of MSIII tended to be higher than BD, and their proportion of lipofuscin deposits tended to be lower, though there were no differences in the quadriceps fiber evaluation.

Conclusions: This study provides the first evidence in humans about the effects of different modalities of MV (controlled, assisted and spontaneous) on diaphragm myofiber damage showing that diaphragm inactivity during mechanical ventilation is associated with the development of VIDD.

\title{
Keywords
}

Ventilator-induced Diaphragm Dysfunction or damage (VIDD), Atrophy, Mechanical Ventilation, Brain Death, Maastricht III, Muscle dysfunction. 


\section{Introduction}

Most critically ill patients admitted to the Intensive Care Unit (ICU) require mechanical ventilation (MV) due to respiratory failure. MV may be associated with adverse effects on respiratory muscles, and disuse atrophy may be the most important mechanism in patients under controlled mechanical ventilation (CMV) [1]. During CMV, the electromyographic activity of the respiratory muscle fibers is diminished or may even stop [2], resulting in a rapid development of respiratory muscle dysfunction, especially diaphragm weakness. Ventilator-induced diaphragm dysfunction (VIDD) is defined as the loss of the diaphragm's capacity to generate force, together with muscle injury and fiber atrophy, and the same acronym has been used before to describe ventilator-induced diaphragm damage [3]. Both VIDD are associated particularly with the use of MV, typically after periods of CMV [4,5,6].

VIDD may play a key role in the pathophysiological mechanisms that make it difficult to wean critically ill patients off MV. It causes direct systemic and pulmonary complications and may exacerbate the patient's comorbidities, thus extending hospital stay. It is also associated with important functional limitations after hospital discharge, reduced quality of life, significantly increased mortality [7] and an increase in costs for the National Health System [8].

Multiple studies with animal [9-15] and human [16-20] models have studied the pathophysiology of VIDD and the molecular pathways involved. The inactivity of the diaphragm caused by MV triggers a state of mitochondrial vulnerability that produces an enzymatic deficiency [10,19], generating excessive reactive oxygen species production and reducing antioxidant activity [14]. It activates apoptotic [12,16] and proteolytic pathways $[9,10,11,14,17,20]$ and promotes the accumulation of lipids in diaphragm due to an excess of energy substrate [18]. It also leads to atrophy of the respiratory muscles and reduces their force-generation capacity.

The loss of muscle strength occurs quickly in some animal models under CMV (6h), with muscle atrophy appearing later (12-18h) [13]. However, other animal studies show fast and complete diaphragmatic recovery from VIDD within 24 hours after returning to spontaneous breathing when CMV is removed before $12 \mathrm{~h}[21,22]$. As in animal models, the establishment of VIDD in humans occurs very quickly $(<24 \mathrm{~h})$, and it increases in severity over time [5].

VIDD severity may be related to several factors that are important for the development of new therapeutic strategies to decrease diaphragm damage. Animal studies [23-28] have shown that spontaneous mechanical ventilation (SMV) may provide more protection than CMV. To date, however, these data have not been confirmed in humans due to the difficulty of obtaining diaphragm biopsies in ventilated critically ill patients; the human data available come from mechanically ventilated brain-dead (BD) organ donors undergoing CMV but they are not representative of ICU patients due to multiple limitations. Recently, in some European countries, donors after circulatory death (DCD) have been accepted for donation and transplantation activity. Maastricht category III donors (MSIII) are DCD in whom cardiac arrest follows planned, controlled withdrawal of life-sustaining treatments. These donors can undergo SMV, but the histological characteristics of their diaphragm have not been evaluated to date.

This is the first study to assess the histological characteristics of the diaphragm in a new human physiopathological model in critical care - MSIII donors - and to compare them with the same data from BD donors. Our hypothesis was that MSIII donors who are able to stimulate their diaphragm may present 
less VIDD than BD who cannot. The main objective was to compare the histological characteristics of MSIII and BD donors ventilated with different forms of MV in respiratory and peripheral muscles.

\section{Methods}

See also the additional information in the online supplement.

\section{Subjects}

MSIII donors, BD donors, and control subjects (CTL) undergoing resection of located pulmonary nodules were included. All patients included were clinically stable at the time of the study; CTL subjects had no episodes of exacerbation or oral steroid treatment in the previous four months. Exclusion criteria included age younger than 18, chronic respiratory failure, treatment with oral steroids, chronic metabolic diseases, suspected para-neoplastic or myopathic syndromes and/or treatment with drugs known to alter muscle structure and/or function. Protocols were approved by the Ethics Committees of Hospital del Mar. All biopsies were performed after written informed consent was obtained.

\section{Biopsies}

All diaphragm biopsy specimens were obtained from the anterior costal diaphragm, lateral to the insertion of the phrenic nerve. All quadriceps biopsies were obtained from the vastus lateralis. All muscle samples were immediately immersed in an alcohol-formol bath and then embedded in paraffin. These tissues were used for the histological analysis.

\section{Clinical data}

Age, sex and body mass index were recorded from all subjects. In both donor groups, reason for donation, severity scores on admission (Acute Physiology and Chronic Health Evaluation II or APACHE II and Sequential Organ Failure Assessment or SOFA), comorbidities, previous treatments, treatments received in the ICU, complications during ICU stay and vital signs previous to surgery were registered. In MSIII donors, the type of preservation (extracorporeal membrane oxygenation (ECMO), cold perfusion or rapid extraction) was also recorded.

The following data on the MV received were recorded: period of time on MV, time on CMV, time on SMV, time with diaphragm stimuli (hours on CMV with Real Respiratory Rate (RRR) above the predetermined Respiratory Rate (PRR) + hours in SMV) and without diaphragm stimuli (hours on CMV with same RRR and PRR) in hours and percentage of time with diaphragm stimuli [(period of time with diaphragm stimuli/hours on MV)*100]. Definitions for these variables are shown in Table 1 (Online Supplement).

\section{Experimental analyses}

The analysis was carried out in the two muscles of both donor groups and CTL. Fiber cross-sectional area (CSA), mean least diameter, and proportions of myofibers expressing myosin heavy chain (MHC) I (type I) or MHCII (type II) were assessed by immunohistochemical analysis under light microscopy (Olympus, Series BX50F3, Olympus Optical Co., Hamburg, Germany). Muscle structure abnormalities (a surrogate 
of muscle damage) were evaluated by previously published methodologies $[29,30]$. The proportion of abnormal muscle (Table 2 Online Supplement) in paraffined samples stained with hematoxylin-eosin was determined by means of the same optic microscopy.

\section{Statistics}

Following the Kolmogorov-Smirnoff test results, continuous variables were expressed as means and standard deviation (SD) when the data were normally distributed or as medians and interquartile range (IR, 25-75\%) otherwise, and categorical variables as frequencies and percentages. Chi-square, Fisher's test, Student's t-test and Mann Whitney U test were used. Comparisons between groups were performed through One-way ANOVA with post hoc Bonferroni test for intragroup comparisons. Relationships between MV duration and CSA were assessed with the Spearman's Rho correlation coefficient. Statistical significance was established at $\mathrm{p}<0.05$. The data were analysed using the statistical package for social sciences 15.0 BM® SPSS Statistics ${ }^{\circledR}$, Chicago, IL, USA) for Windows.

\section{Results}

See also the additional information in the online supplement.

\section{Characteristics of patients}

Thirty-five mechanically ventilated patients (16 MSIII, 14 BD, and 5 CTL) were prospectively included in the study. MSIII were older than BD but not older than CTL (though this may have been due to the small sample size), and there were no significant differences in sex and body mass index among the three groups (Table 1). Clinical characteristics of both donor groups are shown in Table 2. Briefly, the groups did not present differences regarding the severity scores at admission (APACHE II and SOFA), the percentage of comorbidities or treatment prior to admission. During the ICU stay, BD donors received more neuromuscular blockers (NMB, cisatracurium in all cases) and fewer benzodiazepines, opioids and enteral feeding (and for a shorter time) than MSIII donors.

Ventilatory characteristics of donor groups are shown in Table 1. MSIII donors had longer ICU stay with more hours on MV, CMV and SMV than BD. MSIII donors also spent more hours (and a higher percentage of time) with diaphragm stimuli than BD donors.

\section{Immunohistochemical data}

The size of type I and type II fibers was significantly reduced in the diaphragm and quadriceps of both donor groups in comparison with CTL. There were no differences in the MHCI and MHCII CSA of diaphragm between MSIII and BD donors but quadriceps MHCI and MHCII were significantly smaller in MSIII than in BD. These results are shown in Figure 1. The proportions of isoforms did not differ between the three groups (Table 3 Online Supplement).

CSA of diaphragm MHCI and MHCII was lower in MSIII donors who spent 100 or more hours on CMV without diaphragm stimuli than in MSIII donors who spent less than 100h without it. These differences did not appear in quadriceps CSA of the same patients (Figure 2). This threshold was used because 100 hours was the median of hours in CMV without diaphragm stimuli in the MSIII group (Table 1). 
Representative cases of diaphragm and quadriceps-biopsy specimens are shown in Figures 1 and 2 of the online supplement.

MHCI and MHCII diaphragm and quadriceps CSA in all ventilated subjects presented a significantly moderate positive correlation with the time spent in CMV without diaphragm stimulation (Figure 3 Online Supplement). Diaphragm atrophy seemed to appear sooner under CMV without diaphragm stimulation than quadriceps atrophy (100 hours vs 150 hours), but no significant differences were found (Figure 4 Online Supplement).

Differences between MSIII with cold perfusion and rapid extraction are shown in the Online Supplement (Table 4). There were no differences in the time under MV, but donors with cold perfusion preservation spent more hours under CMV and without diaphragm stimuli than donors with rapid extraction. Cold perfusion presented smaller fibers in diaphragm MHCII than rapid extraction without differences in any other clinical variables.

\section{Muscle structure abnormalities}

The histological evaluation of muscle structure abnormalities showed an increase in abnormal fibers in diaphragms of MSIII and BD compared with CTL, although the difference was not statistically significant (possibly due to the small sample size). Diaphragms of BD had a higher proportion of lipofuscin deposits than CTL; this proportion tended to be higher in MSIII, though the difference did not reach statistical significance. Both donor groups presented decreased internal nuclei compared to CTL. Proportion of internal nuclei in diaphragms of MSIII tend to be higher than BD and their proportion of lipofuscin deposits tended to be lower. There were no significant differences in the fiber evaluation in quadriceps between the three groups (Table 3); nor were there significant differences in other categories assessed in diaphragms. Several representative images are shown in the online supplement (Figure 5 and 6).

\section{Discussion}

This is the first study with human samples to analyse the histological characteristics of respiratory (diaphragm) and peripheral (quadriceps) muscles in two types of organ donor (MSIII and BD) undergoing different forms of MV (SMV and CMV). The results show that MSIII who have the capacity to stimulate their diaphragm present less VIDD than BD.

Until now, human data related to VIDD are basically available from two groups of patients. The bulk of the evidence derives from mechanically ventilated BD donors; only very recently have studies expanded to include mixed ICU populations with underlying diseases. Levine et al [31] were the first to demonstrate that complete diaphragmatic inactivity and MV result in atrophy of human diaphragm myofibers in BD. But studies with BD donors present a clear limitation, given that brain death itself can generate multiple systemic changes, and is thus an important confounder [32-35]. BD donors do not exhibit the clinical features of critically ill patients; they are distinguished by the complete absence of neural activation of the diaphragm, metabolic stress, and brain ischemia [32-35]. Recent studies in mixed ICU populations present an important limitation due to the difficulty of obtaining diaphragmatic samples that allow the performance of histological studies [20]. A relevant advance in the present study is our use of a novel pathophysiological model, MSIII organ donors, and our assessment of their diaphragmatic 
structure. This recently described donor profile [36] could present multiple advantages over the groups used in previous models.

First, MSIII are critically ill patients with irreversible severe disease in whom the decision is taken to withdraw life support measures in a controlled manner. This means that they are a highly representative population of critically ventilated patients. MSIII presented a longer ICU stay with a fewer need for NMB and a higher need for sedation and opioids than $\mathrm{BD}$ and also had more infectious complications. With regard to these differences, anesthetics can be ruled out as causes of VIDD, since studies using appropriate controls concluded that the decreased contractility was due to the effects of MV per se [37]. The decreased contractility secondary to CMV cannot be attributed solely to NMB, since it was also observed in studies that did not use these drugs. However, the effects of $24 \mathrm{~h}$ of CMV and aminosteroidal NMB (rocuronium) are synergistic in depressing diaphragm contractility, in inducing atrophy and in upregulating proteolysis, a synergism not observed with different doses of benzylisoquinoline NMB (cisatracurium) [37].

Second, among other differences with respect to BD, in most cases MSIII were able to spontaneously stimulate their diaphragm and tolerated SMV well, since they spent more hours under SMV and with diaphragm stimuli.

Another strength of this study is the analysis of two muscles, one respiratory (diaphragm) and the other non-respiratory (quadriceps). As we expected, both presented significant CSA reductions in MSIII and in BD compared to CTL. It is known that in limb muscles, contractile activity regulates protein turnover and muscle mass, and muscle disuse accelerates protein degradation [38]. MV may impose bed rest and may indirectly prolong immobility as a result of the use of sedatives or NMB, which may promote neuropathy, muscle wasting, and loss of muscle function in non-respiratory muscles. Indeed, our results showed that quadriceps of MSIII patients, who spent more days intubated and immobilized in the ICU with greater need of these drugs, presented a decreased fiber size than BD. Similarly, Powers and colleagues [39] recently speculated that the sudden creation of a state of diaphragm disuse during "full support" MV is the key initiator of the cascading changes that lead to VIDD. But while the diaphragm is characterized by a permanent cyclic activity, normal limb muscles are not constantly active. Furthermore, although both are striated muscles, their fiber type composition differs, as shown both by our results and by previous reports $[40,41]$.

Animal experiments in recent years have demonstrated that increasing diaphragm muscle contraction by use of assisted modes during MV may slow the evolution of VIDD [23-28]. On the one hand, in rats, rabbits and piglets, partial support MV has been shown to prevent protease activation in the diaphragm [23], the increased expression of ubiquitin-proteasome system components and diaphragm atrophy, and maintains contractile function [24-26]. On the other, reducing the level of support also protects rat diaphragm against oxidative stress and atrophy [27]. Finally, intermittent spontaneous breathing in ventilated rats minimizes the reduction in diaphragm force and fiber size during MV [28].

As we expected, our results regarding muscle atrophy and diaphragm abnormalities in humans are in accordance with these data observed in animal models. First of all, our results showed that BD and MSIII donors presented major diaphragmatic atrophy in both types of muscle fibers, slow-twitch (type I or expressing MHCI) and fast-twitch (type II or expressing MHCII) after a variable long-term period of ventilation compared to CTL. Second, there were no differences between diaphragm CSA in MHC I or 
MHC II in MSIII compared to BD, although MSIII donors spent significantly longer under MV and CMV. In addition, MSIII donors spent more hours under SMV and a higher proportion of time with diaphragm stimuli. Moreover, when MSIII donors were divided into two groups according to the number of hours without diaphragm stimuli, it was found that diaphragm CSA of MSIII donors with more hours of ventilation without diaphragm stimuli were smaller than those with fewer hours without diaphragm stimuli. So it seems that MSIII donors present a protective factor against the development of respiratory muscle atrophy which could be attributed to the higher rate of SMV used and the diaphragm stimulation maintained during MV.

At present there are no data regarding other morphological muscle abnormalities existing in the respiratory muscles of ventilated patients. Our results showed that diaphragm of MSIII had a lower percentage of lipofuscin and a higher percentage of internal nuclei than diaphragm of BD, and there were no differences in peripheral muscles in any of the groups analysed. The accumulation of lipofuscin indicates more remains of lipid destruction, which is an indirect marker of oxidative stress and may indicate a more advanced stage of muscle protein degradation in $\mathrm{BD}$. These findings are in accordance with previous reports indicating that $\mathrm{CMV}$ induces the production of reactive oxygen species in the diaphragm, which is required for the activation of several key proteolytic systems involved in MVinduced diaphragmatic atrophy [2]. The lesser appearance of internal nuclei indicates a lower capacity of muscle repair in the diaphragm of the same patients. These results suggest that respiratory muscles of MSIII donors are less damaged than those from BD, and once again this may be due to a protective factor induced by diaphragmatic stimulation. Although increases in the proportion of necrotic fibers in the diaphragm of some ventilated patients have been reported in the literature [42,43], these studies analysed septic patients in whom VIDD and sepsis-induced diaphragm dysfunction (SIDD) may have coexisted. This combination of sepsis and ventilation could have an additive (if not synergic) deleterious impact on diaphragm function, but was not present in our series or in the BD series described by Levine [31].

A variety of interventions may be useful in preventing VIDD. Muscle-protective ventilation strategies (proportional assist ventilation and neurally adjusted ventilatory assist) aim to titrate ventilation in order to maintain appropriate levels of inspiratory muscle effort and optimize synchrony between patient and ventilator [44]. Moreover, interventions involving the molecular pathways studied and the reversal of muscle disuse through electrostimulation are under investigation. The time is indeed ripe for clinical trials in this important area, but new tools are also needed that allow us to evaluate respiratory muscle function at the bedside. The current literature suggests that diaphragm ultrasound could be a useful and accurate tool for detecting diaphragmatic dysfunction in critically ill patients, predicting extubation success or failure, monitoring respiratory workload, and assessing atrophy in patients who are mechanically ventilated [45].

\section{Study limitations}

First of all, the groups compared contained different kinds of patients (BD and MSIII). However, the aim of this study was to present the first assessment of the histological characteristics of MSIII patients, and to compare them with those of $\mathrm{BD}$, the only previously studied population. Neither group was pure in terms of the ventilatory methods used, so it is difficult to attribute the changes described to the type of ventilation received. To minimize this heterogeneity, in this study the hours under CMV and SMV were 
carefully counted as well as the difference between the PRR and RRR in order to calculate the time under MV in which patients stimulated or did not stimulate their diaphragm. These data are detailed in table 2. Moreover, inside the MSIII group we also compared those who spent more hours on CMV with those under fewer hours of CMV, and cold perfusion versus rapid extraction. Further studies will be necessary to compare pure groups. Another limitation is that we cannot exclude the possibility that factors other than MV per se were involved in the histological alterations found in both donor groups. We eliminated patients suffering from other conditions known to alter respiratory muscle function in our study, and we recorded the treatments known to be deleterious. We also included a limb muscle as internal control, subjected to the same systemic factors as the diaphragm (except for MV) in order to assess whether the changes were due to the different forms of ventilation used. Likewise - and this is a major limitation since our data are histological we can only speculate about their functional significance and we can only refer to ventilator-induced diaphragm damage. Further studies will be necessary to elucidate why diaphragmatic rest reduces diaphragm CSA, and how preserving a certain degree of spontaneous diaphragm contractile activity protects against diaphragm atrophy.

\section{Conclusions}

This study provides the first evidence in humans about the effects of different modalities of MV (controlled, assisted and spontaneous) on diaphragm myofiber damage showing that diaphragm inactivity during mechanical ventilation is associated with the development of VIDD. It suggests that the deleterious effects of diaphragm unloading generated by CMV in humans can be attenuated by maintaining a certain level of respiratory muscle stimulation during MV. Future studies using MSIII donor models should include more decisive measures of protein turnover and redox balance along with diaphragmatic contractile measurements in these patients.

\section{Take Home Message}

Maastricht III donors present less ventilator-induced diaphragm damage (VIDD) than brain-dead donors, probably due to their ability to stimulate their diaphragm. In this regard, we recommend that the period of controlled mechanical ventilation in critically ill ventilated patients should be kept short and that ventilation modes with sustained patient effort should be introduced promptly.

\section{Acknowledgments}

We thank Marta Gas for her administrative and technical support, the transplant coordination nursing team of the Hospital del Mar for their help obtaining with the sample and Judit Boncompte for her help with the experimental analyses.

\section{Author contributions}

JMC, ID, JRM conceived and designed the study.

JMC, ID, MB, LC, AZ, MPG, SPG, CV, PPT and AC contributed to data acquisition and analysis. 
JMC, ID, SPG, JG, JRM made important intellectual contributions and actively participated in the interpretation of the data and wrote the paper.

All authors contributed to critical examination of the paper for important intellectual content and approval of the final manuscript.

\section{Conflicts of interests}

All named authors declare that they have no conflicting interests.

\section{Funding and Role of the sponsors}

This study has been supported by Spanish Critical Patient Foundation (FEEC 2017) and Catalan Society of Critical Care (SOCMIC 2018). The study sponsors had no role in the study design, data collection, data analysis, data interpretation, or writing of the report. The corresponding author (JMC) had full access to all the data in the study and final responsibility for the decision to submit for publication.

\section{Ethics Committee Approval}

The institutional review board of PSMAR approved the original study (IRB Ref 2017/7183/I).

\section{References}

1.- Dres M, Goligher EC, Heunks LMA, Brochard LJ (2017) Critical illness-associated diaphragm weakness. Intensive Care Med 43(10):1441-1452.

2.- Huibin Tang, Joseph B Shrager (2018) Translational review: The signaling Network Resulting in Ventilator-induced diaphragm dysfunction. Am J Respir Cell Mol Biol. In press. doi: 10.1165/rcmb.2018$0022 \mathrm{TR}$.

3.- Li LF, Tien ML, Leung SY, Lin MC (2011) High tidal volume mechanical ventilation elicits increased activity in protein kinase $\mathrm{B}$ and c-Jun $\mathrm{NH}_{2}$-terminal kinase pathways in mouse diaphragm. Intensive Care Med 37:2015-2022.

4.- Vassilakopoulos T, Petrof BJ (2004) Ventilator-induced diaphragmatic dysfunction. Am J Respir Crit Care Med 169:336-341.

5.- Jaber S, Petrof BJ, Jung B, Chanques G, Berthet JP, Rabuel C, et al (2011) Rapidly progressive diaphragmatic weakness and injury during mechanical ventilation in humans. Am J Respir Crit Care Med 183(3):364-71.

6.- Petrof B, Hussain S (2016) Ventilator-induced diaphragmatic dysfunction: what have we learned? Curr Opin Crit Care 22(1):67-72.

7.- Supinski GS, Ann Callahan L (2013) Diaphragm weakness in mechanically ventilated critically ill patients. Crit Care 17(3):R120.

8.- Herridge MS, Tansey C, Matté A, Tomlinson G, Diaz-Granados N, Cooper A, et al (2009) Functional Disability 5 Years after Acute Respiratory Distress Syndrome. N Engl J Med 364(14):123-34.

9.- Smuder AJ, Nelson WB, Hudson MB, Kavazis AN, Powers SK (2010) Inhibition of the ubiquitinproteasome pathway does not protect against ventilator-induced accelerated proteolysis or atrophy in the 
diaphragm. Surg Clin North Am 90(5):955-68.

10.- Smuder AJ, Hudson MB, Nelson WB, Kavazis AN, Powers SK (2012) Nuclear factor- $\kappa B$ signaling contributes to mechanical ventilation-induced diaphragm weakness. Crit Care Med 40(3):927-34.

11.- Smuder AJ, Sollanek KJ, Min K, Nelson WB, Powers SK (2015) Inhibition of forkhead boxOspecific transcription prevents mechanical ventilation-induced diaphragm dysfunction. Crit Care Med 43(5):e133-42.

12.- Azuelos I, Jung B, Picard M, Liang F, Li T, Lemaire C, et al (2015) Relationship between Autophagy and Ventilator-induced Diaphragmatic Dysfunction. Anesthesiology 122(6):1349-61.

13.- Matecki S, Dridi H, Jung B, Saint N, Reiken SR, Scheuermann V, et al (2016) Leaky ryanodine receptors contribute to diaphragmatic weakness during mechanical ventilation. Proc Natl Acad Sci 113(32):9069-74.

14.- Matecki S, Jung B, Saint N, Scheuermann V, Jaber S, Lacampagne A (2017) Respiratory muscle contractile inactivity induced by mechanical ventilation in piglets leads to leaky ryanodine receptors and diaphragm weakness. J Muscle Res Cell Motil 0(0):1-8.

15.- Jaber S, Sebbane M, Koechlin C, Hayot M, Capdevila X, EledJam J et al (2005) Effects of short vs. prolonged mechanical ventilation on antioxidant systems in piglet diaphragm. Intensive Care Med 31(1427-1433).

16.- Hussain SNA, Mofarrahi M, Sigala I, Kim HC, Vassilakopoulos T, Maltais F, et al (2010) Mechanical ventilation-induced diaphragm disuse in humans triggers autophagy. Am J Respir Crit Care Med 182(11):1377-86.

17.- Levine S, Biswas C, Dierov J, Barsotti R, Shrager JB, Nguyen T, et al (2011) Increased proteolysis, myosin depletion, and atrophic AKT-FOXO signaling in human diaphragm disuse. Am J Respir Crit Care Med 183(4):483-90.

18.- Picard M, Jung B, Liang F, Azuelos I, Hussain S, Goldberg P, et al (2012) Mitochondrial dysfunction and lipid accumulation in the human diaphragm during mechanical ventilation. Am J Respir Crit Care Med 186(11):1140-9.

19.- Tang H, Smith IJ, Hussain SN a, Goldberg P, Lee M, Sugiarto S, et al (2014) The JAK-STAT pathway is critical in ventilator-induced diaphragm dysfunction. Mol Med 20(36):579-89.

20.- Hooijman PE, Beishuizen A, Witt CC, de Waard MC, Girbes ARJ, Spoelstra-de Man AME, et al (2015) Diaphragm muscle fiber weakness and ubiquitin-proteasome activation in critically ill patients. Am J Respir Crit Care Med 191(10):1126-38.

21.- Bruells CS, Bergs I, Rossaint R, Du J, Bleilevens C, Goetzenich A, et al (2014) Recovery of diaphragm function following mechanical ventilation in a rodent model. PLoS One 9(1):3-10.

22.- Callahan LA, Supinski GS (2013) Rapid and complete recovery in ventilator-induced diaphragm weakness-problem solved? J Appl Physiol 115(6):773-4.

23.- Futier E, Constantin JM, Combaret L, Mosoni L, Roszyk L, Sapin V, et al (2008) Pressure support ventilation attenuates ventilator-induced protein modifications in the diaphragm. Crit Care 12(5):R116.

24.- Sassoon CS, Zhu E, Caiozzo VJ (2004) Assist-control mechanical ventilation attenuates ventilatorinduced diaphragmatic dysfunction. Am J Respir Crit Care Med 170(6):626-632. 
25.- Hudson MB, Smuder AJ, Nelson WB, Wiggs MP, Shimkus KL, Fluckey JD, et al (2015) Partial support ventilation and mitochondrial targeted antioxidants protect against ventilator-induced decreases in diaphragm muscle protein synthesis. PLoS One 10(9):e0137693.

26.- Jung B, Constantin JM; Rossel N, Le Goff C, Sebbane M, Coisel Y, et al (2010) Adaptive support ventilation prevents ventilator-induced diaphragmatic dysfunction in piglet: an in vivo and in vitro study. Anesthesiology 112(6):1435-1443.

27.- Hudson MB, Smuder AJ, Nelson WB, Bruells CS, Levine S, Powers SK (2012) Both High Level Pressure Support Ventilation and Controlled Mechanical Ventilation Induce Diaphragm Dysfunction and Atrophy. Crit Care Med 40(4):1254-1260.

28.- Gayan-Ramirez G, Testelmans D, Maes K, Rácz GZ, Cadot P, Zádor E, et al (2005) Intermittent spontaneous breathing protects the rat diaphragm from mechanical ventilation effects. Crit Care Med 33(12):2804-2809.

29.- Macgowan NA, Evans KG, Road JD, Reid WD (2001) Diaphragm injury in individuals with airflow obstruction. Am J Respir Crit Care Med 163(7):1654-9.

30.- Pascual-Guardia S, Wodja E, Gorostiza A, López de Santamaría E, Gea J, Gáldiz JB, et al (2013) Improvement in quality of life and exercise capacity without muscular biology changes after general training in patients with severe chronic obstructive pulmonary disease. Med Clin 140(5):200-6.

31.- Levine S, Nguyen T, Taylor N, Friscia M, Budak MT, Rothenberg P, et al (2007) Rapid Disuse Atrophy of Diaphragm Fibers in Mechanically Ventilated Humans. N Engl J Med 358(13):1327-35.

32.- Belperio JA, Keane MP, Lynch JP, Strieter RM (2006) The role of cytokines during the pathogenesis of ventilator-associated and ventilator-induced lung injury. Semin Respir Crit Care Med 27:350-64.

33.- Amado JA, López-Espadas F, Vázquez-Barquero A, Salas E, Riancho JA, López-Cordovilla, et al (1995) Blood levels of cytokines in brain-dead patients: relationship with circulating hormones and acutephase reactants. Metabolism 44:812-16.

34.- Auraen H, Molines TE, Bjortuft O, Bakkan PA, Geiran O, Kongerud J, et al (2013) Multiorgan procurement increases systemic inflammation in brain dead donors. Clin Transplant. 27(4):613-8.

35.- Barklin A (2009) Systemic inflammation in the brain-dead organ donor. Acta Anaesthesiol Scand 53: 425-435, 2009.

36.- Rubio JJ, Palacios D (2016) Reflections upon donation after controlled cardiac death (Maastricht type III donors). Med Intensiva 40(7):431-433.

37.- Vassilakopoudos T (2008) Ventilator-induced diaphragm dysfunction: the clinical relevance of animal models. Intensive Care Med 34:7-16.

38.- Latronico N, Herridge M, Hopkins R, Angus D, Hart N, Hermans G, et al (2017) The ICM research agenda on intensive care unit-acquired weakness. Intensive Care Med 43(9):1270-1281.

39.- Powers SK, Smuder AJ, Fuller D, Levine S (2013) Crosstalk proposal: Mechanical ventilationinduced diaphragm atrophy is primarily due to inactivity. J Physiol 591(21):5255-5257.

40.- Polla B, D’Antona G, Bottinelli R, Reggiani C (2004) Respiratory muscle fibres: specialisation and plasticity. Thorax 59:808-817. 
41.- Barreiro E, Ferrer D, Sanchez F, Minguella J, Marin-Corral J, Martinez-Llorens J, et al (2011) Inflammatory cells and apoptosis in respiratory and limb muscles of patients with COPD. J Appl Physiol 111(3):808-17.

42.- Latronico N, Fenzi F, Recupero D, Guarneri B, Tomelleri G, Tonin P, et al (1996) Criticcal illness myopathy and neuropathy. Lancet 347(9015):1579-82.

43.- Puthucheary ZA, Rawal J, McPhail M, Connolly B, Ratnayake G, Chan P, et al (2013) Acute skeletal muscle wasting in critically illness. JAMA 310(15):1591-600.

44.- Pettenuzzo T, Fan E (2017) 2016 Year in Review: Mechanical Ventilation. Respir Care 62(5):629635.

45.- Zambon M, Greco M, Bocchino S, Cabrini L, Beccaria PF, Zangrillo A (2017) Assessment of diaphragmatic dysfunction in the critically ill patient with ultrasound: a systematic review. Intensive Care Med 43(1):29-38 . 


\section{Figure Legends}

A)

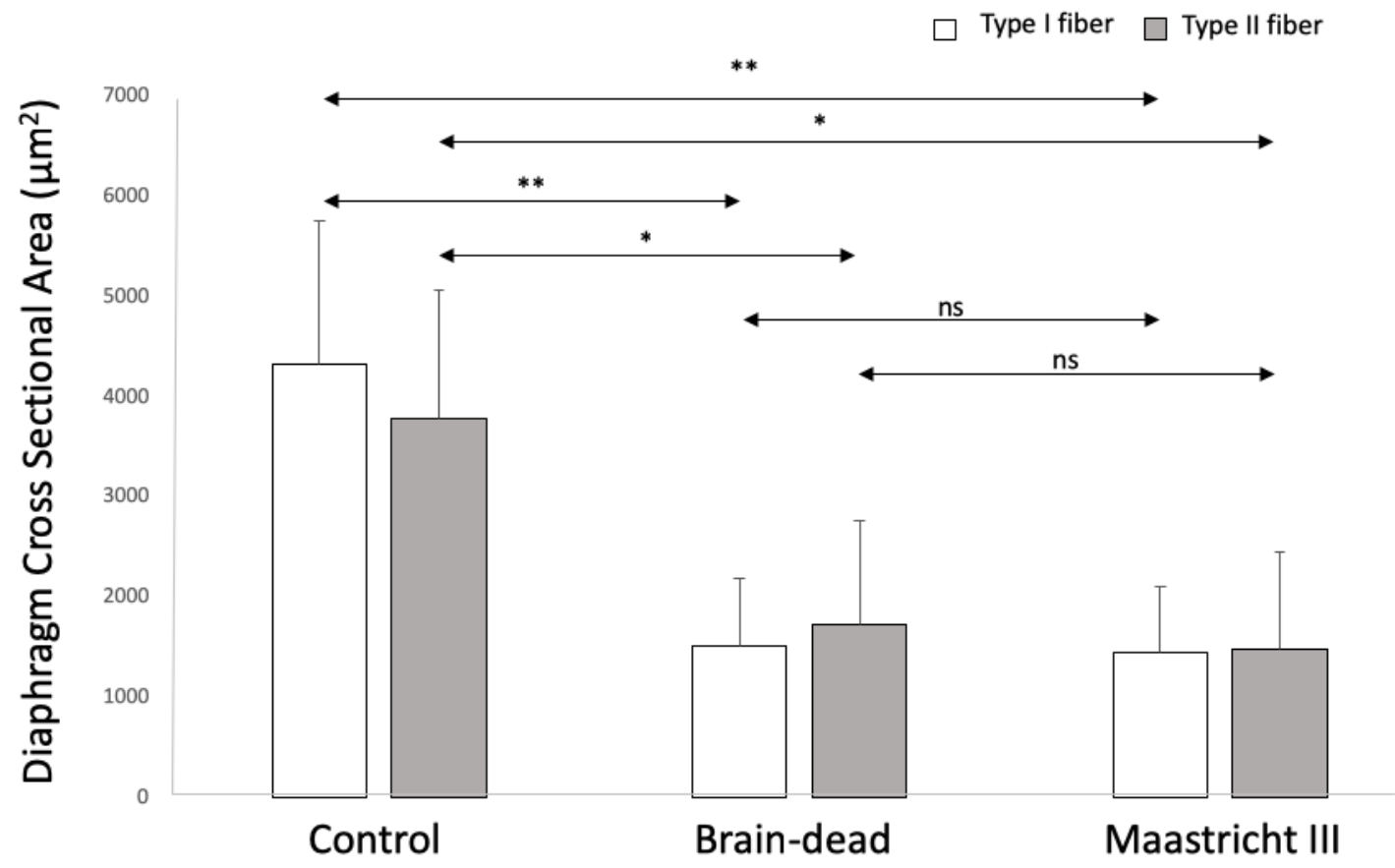

B)

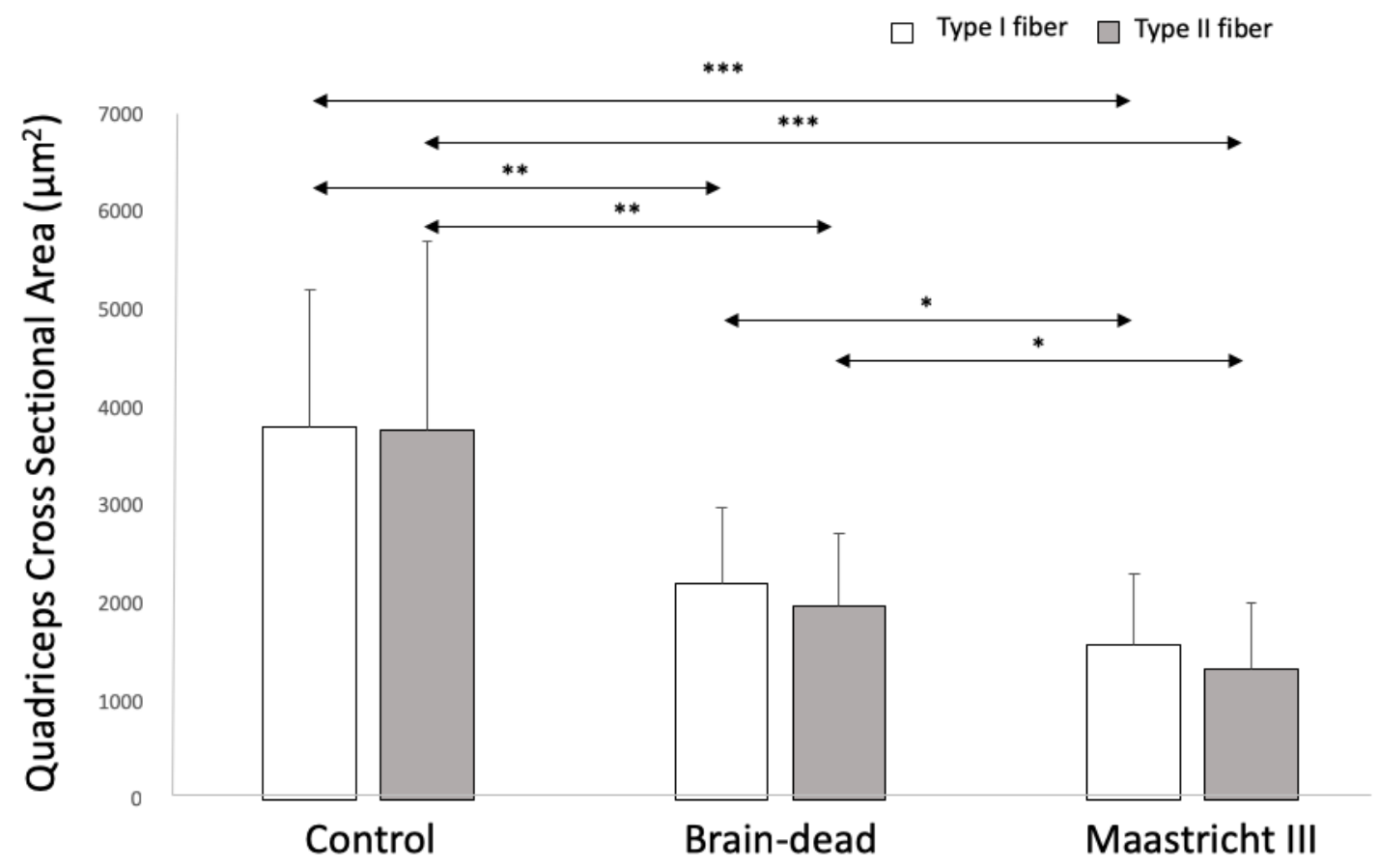

Figure 1. Cross sectional area of $\mathrm{MHCl}$ (type I) and $\mathrm{MHCll}$ (type II) fibers of diaphragm (A) and quadriceps (B) in ventilated patients. ${ }^{*} p<0.05 ;{ }^{* *} p<0.01 ;{ }^{* * *} p<0.001$ 
A)

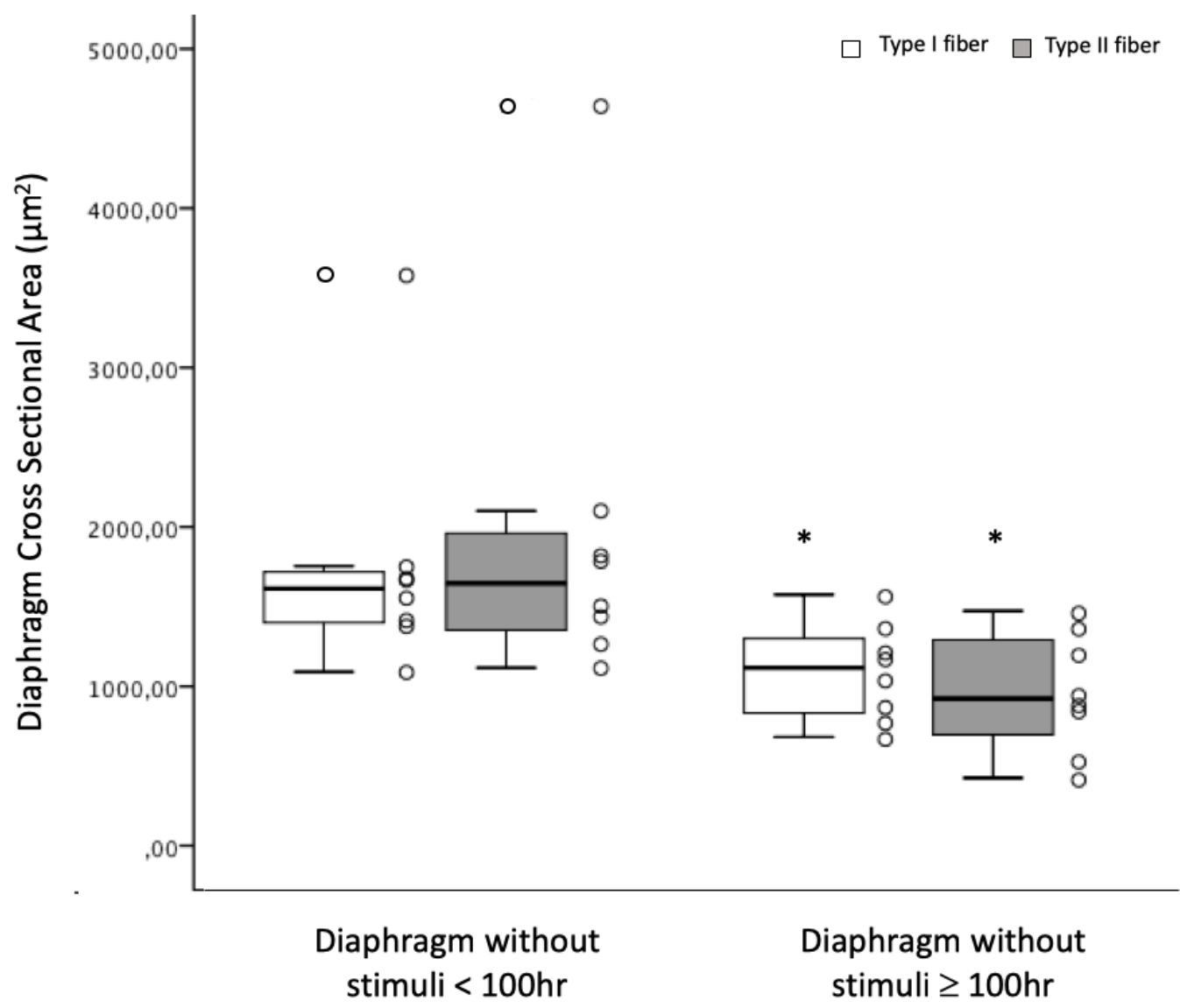

B)

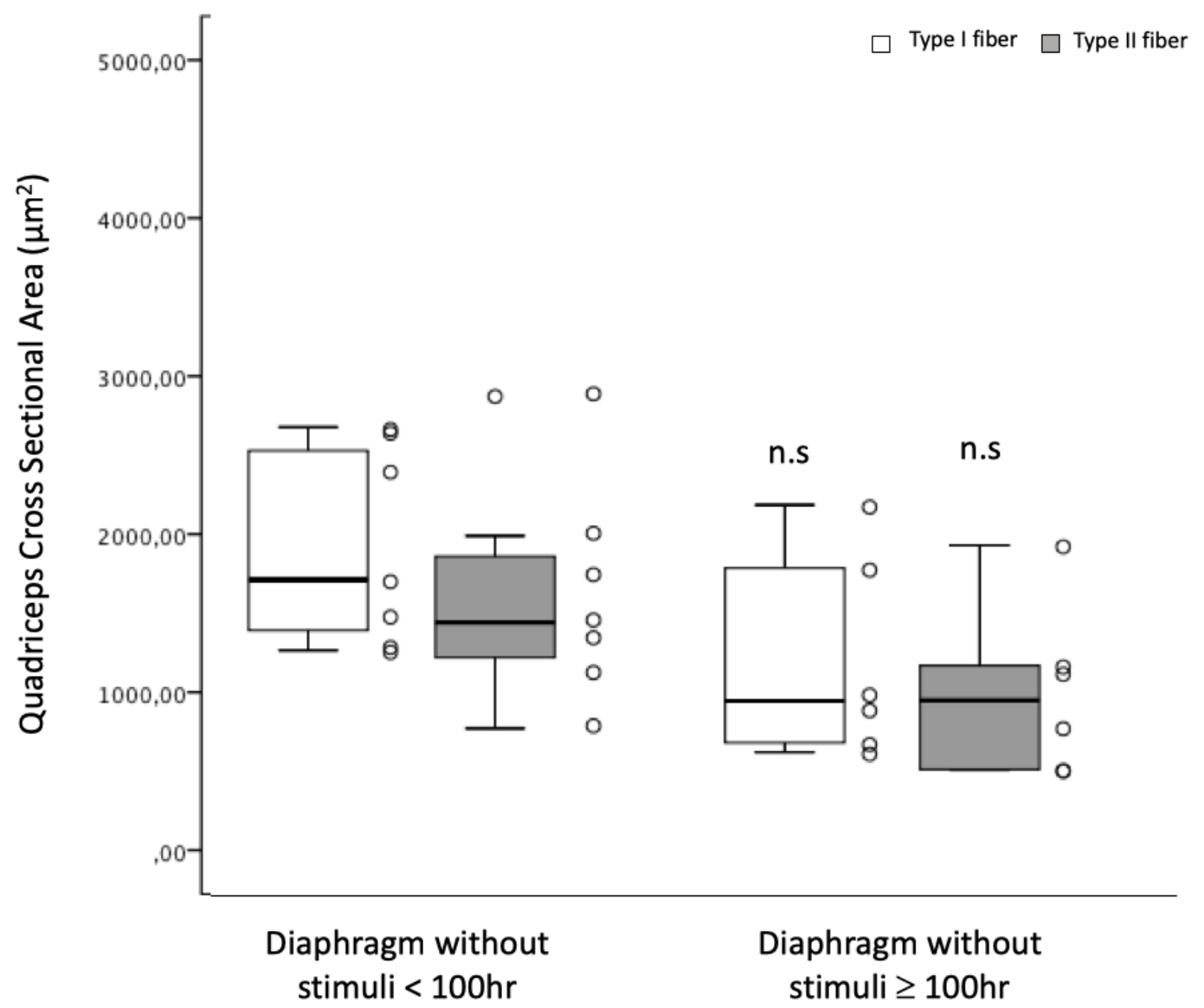


Figure 2. Cross sectional area of $\mathrm{MHCl}$ (type I) and $\mathrm{MHCll}$ (type II) fibers of diaphragm (A) and quadriceps (B) in ventilated patients without DIAPHRAGM stimuli. * $p<0.05$ 


\begin{tabular}{|c|c|c|c|c|c|c|c|c|c|c|c|c|}
\hline Subject & Cause of donation & $\begin{array}{c}\text { Type of } \\
\text { donation }\end{array}$ & $\begin{array}{l}\text { Age } \\
\text { (yr) }\end{array}$ & $\begin{array}{c}\text { Sex } \\
\text { (male) }\end{array}$ & $\begin{array}{c}\text { BMI } \\
\left(\mathrm{kg} / \mathrm{m}^{2}\right)\end{array}$ & $\begin{array}{l}\text { MV } \\
\text { (h) }\end{array}$ & $\begin{array}{c}\text { Total CMV } \\
\text { (h) }\end{array}$ & $\begin{array}{c}\text { CMV with RRR=PRR (DF } \\
\text { without stimuli, h) }\end{array}$ & $\begin{array}{l}\text { CMV with } \\
\text { RRR>PRR (h) }\end{array}$ & $\begin{array}{c}\text { Total SMV } \\
\text { (h) }\end{array}$ & $\begin{array}{c}\text { DF stimuli } \\
\text { (h) }\end{array}$ & $\begin{array}{c}\text { DF stimuli } \\
\text { (\%) }\end{array}$ \\
\hline & & & & & & A & B & B1 & B2 & c & $B 2+C$ & $([\mathrm{~B} 2+\mathrm{C}) / \mathrm{A}] * 100$ \\
\hline \multicolumn{13}{|l|}{ Control } \\
\hline 1 & & & 61 & $\mathrm{~F}$ & 25 & 5 & 5 & 5 & 0 & 0 & 0 & 0 \\
\hline 2 & & & 61 & $M$ & 23 & 4 & 4 & 4 & 0 & 0 & 0 & 0 \\
\hline 3 & & & 74 & $M$ & 23 & 4 & 4 & 4 & 0 & 0 & 0 & 0 \\
\hline 4 & & & 63 & $M$ & 27 & 3 & 3 & 3 & 0 & 0 & 0 & 0 \\
\hline \multirow[t]{2}{*}{5} & & & 58 & $M$ & 30 & 4 & 4 & 4 & 0 & 0 & 0 & 0 \\
\hline & & & $63(6)$ & $4(80)$ & $26(3)$ & $3(3-4)^{f+r, \ldots \# \#}$ & 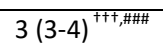 & $3(3-4)^{f+1, \# \# \#}$ & $0(0-0)^{\#}$ & $0(0-0)^{\# \#}$ & $0(0-0)^{\# \# \#}$ & $0(0-0)^{\# \# \#}$ \\
\hline \multicolumn{13}{|l|}{ Brain Dead } \\
\hline 1 & Cardiac arrest & & 66 & $M$ & 36 & 63 & 63 & 63 & 0 & 0 & 0 & 0 \\
\hline 2 & Cardiac arrest & & 59 & $M$ & 28 & 117 & 92 & 92 & 0 & 25 & 25 & 21,37 \\
\hline 3 & Cardiac arrest & & 33 & $\mathrm{~F}$ & 21 & 46 & 46 & 43 & 3 & 0 & 3 & 6,52 \\
\hline 4 & Cerebrovascular accident & & 81 & $M$ & 26 & 29 & 26 & 26 & 0 & 3 & 3 & 10,34 \\
\hline 5 & Cardiac arrest & & 30 & $M$ & 24 & 97 & 74 & 67 & 7 & 23 & 30 & 30,93 \\
\hline 6 & Cerebrovascular accident & & 59 & $M$ & 21 & 7 & 7 & 7 & 0 & 0 & 0 & 0 \\
\hline 7 & Cerebrovascular accident & & 75 & $M$ & 21 & 13 & 13 & 13 & 0 & 0 & 0 & 0 \\
\hline 8 & Cardiac arrest & & 40 & $M$ & 25 & 54 & 54 & 53 & 1 & 0 & 1 & 1,85 \\
\hline 9 & Cerebrovascular accident & & 75 & $\mathrm{~F}$ & 29 & 54 & 54 & 54 & 0 & 0 & 0 & 0 \\
\hline 10 & Cerebrovascular accident & & 44 & $M$ & 28 & 120 & 83 & 66 & 17 & 37 & 54 & 45 \\
\hline 11 & Cerebrovascular accident & & 65 & $M$ & 28 & 79 & 79 & 79 & 0 & 0 & 0 & 0 \\
\hline
\end{tabular}




\begin{tabular}{|c|c|c|c|c|c|c|c|c|c|c|c|}
\hline 12 & Meningitis & 32 & $\mathrm{M}$ & 24 & 138 & 138 & 138 & 0 & 0 & 0 & 0 \\
\hline 13 & Cardiac arrest & 73 & $\mathrm{M}$ & 29 & 147 & 108 & 105 & 3 & 39 & 42 & 28,57 \\
\hline 14 & Severe head trauma & 51 & $\mathrm{~F}$ & 31 & 238 & 238 & 238 & 0 & 0 & 0 & 0 \\
\hline & & 56 (17) & $11(78)$ & $27(4)$ & 71 (42-124) & $68(41-96)$ & 64 (39-95) & $0(0-3)$ & $0(0-24)$ & $0(0-26)$ & $1(0-23)$ \\
\hline
\end{tabular}

\section{MaastrichtIII}

\begin{tabular}{|c|c|c|c|c|c|c|c|c|c|c|c|c|}
\hline 1 & Cardiac arrest & RE & 47 & $\mathrm{M}$ & 22 & 126 & 29 & 25 & 4 & 97 & 101 & 80,16 \\
\hline 2 & Cardiac arrest & $\mathrm{CP}$ & 49 & $\mathrm{~F}$ & 21 & 196 & 134 & 134 & 0 & 62 & 62 & 31,63 \\
\hline 3 & Cerebrovascular accident & RE & 77 & $\mathrm{~F}$ & 38 & 22 & 22 & 22 & 0 & 0 & 0 & 0 \\
\hline 4 & Meningitis & RE & 72 & M & 25 & 208 & 75 & 59 & 16 & 133 & 149 & 71,63 \\
\hline 5 & Cerebrovascular accident & RE & 78 & $\mathrm{~F}$ & 22 & 382 & 51 & 49 & 2 & 331 & 333 & 87,17 \\
\hline 6 & Cerebrovascular accident & $\mathrm{RE}$ & 67 & M & 24 & 381 & 108 & 58 & 50 & 273 & 323 & 84,78 \\
\hline 7 & Cardiac arrest & RE & 57 & $\mathrm{~F}$ & 31 & 352 & 352 & 213 & 139 & 0 & 139 & 39,49 \\
\hline 8 & Cerebrovascular accident & $\mathrm{CP}$ & 63 & M & 22 & 110 & 110 & 110 & 0 & 0 & 0 & 0 \\
\hline 9 & Severe head trauma & ECMO & 73 & M & 30 & 97 & 97 & 73 & 24 & 0 & 24 & 24,74 \\
\hline 10 & Cardiac arrest & $\mathrm{CP}$ & 73 & $\mathrm{~F}$ & 28 & 560 & 194 & 159 & 35 & 366 & 401 & 71,61 \\
\hline 11 & Severe head trauma & RE & 87 & M & 24 & 236 & 152 & 128 & 24 & 84 & 108 & 45,76 \\
\hline 12 & Cerebrovascular accident & RE & 77 & M & 27 & 156 & 156 & 93 & 63 & 0 & 63 & 40,38 \\
\hline 14 & Cardiac arrest & $\mathrm{RE}$ & 73 & M & 30 & 316 & 182 & 182 & 0 & 134 & 134 & 42,41 \\
\hline 15 & Cardiac arrest & ECMO & 57 & $\mathrm{~F}$ & 27 & 159 & 65 & 35 & 30 & 94 & 124 & 77,99 \\
\hline 16 & Cerebrovascular accident & $\mathrm{CP}$ & 66 & $\mathrm{~F}$ & 29 & 213 & 201 & 197 & 4 & 12 & 16 & 7,51 \\
\hline
\end{tabular}




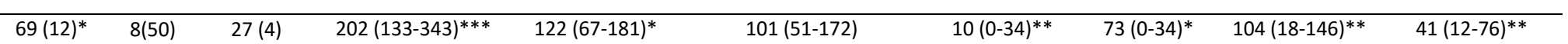

BMI: body mass index; MV: mechanical ventilation; CMV: controlled mechanical ventilation; SMV: spontaneous mechanical ventilation; RRR: real respiratory rate; PRR: predetermined respiratory rate; DF:

diaphragm; F: female; M: male; RE: rapid extraction; CP: cold perfusion; ECMO: extracorporeal membrane oxygenation.

Table 1. Characteristics of organ donors. ${ }^{\text {ft+ }}$ Between Control and Brain Dead, $p<0.001 ;{ }^{\#}$ Between Control and MaastrichtIII, $p<0.05 ;{ }^{\text {\#l }}$ Between Control and MaastrichtIII, $p<0.01 ;$

Between Control and MaastrichtIII, p<0.001. * Between Brain Dead and MaastrichtIII, p<0.05; ** Between Brain Dead and MaastrichtIII, p<0.01; *** Between Brain Dead and MaastrichtIII, $\mathrm{p}<0.001$. Note that hours with DF stimuli $(\mathrm{B} 2+\mathrm{C})$ are the hours of SMV (C) plus the hours in CMV with RRR higher than PRR (B2). Data expressed as mean (SD), median (IQR) or $\mathrm{n}(\%)$. 


\begin{tabular}{|c|c|c|c|}
\hline & $\begin{array}{c}\text { Brain-dead } \\
n=14\end{array}$ & $\begin{array}{l}\text { Maastricht III } \\
n=16\end{array}$ & p-value \\
\hline \multicolumn{4}{|l|}{ Severity scores, mean (SD) } \\
\hline APACHE II score at admission & $29(8)$ & $24(6)$ & $\mathrm{ns}$ \\
\hline SOFA score at admission & $9(3)$ & $7(3)$ & $\mathrm{ns}$ \\
\hline Days of ICU stay, median (IQR) & $4(2-6)$ & $8(5-12)$ & 0.002 \\
\hline \multicolumn{4}{|l|}{ Toxic habits, n (\%) } \\
\hline Smoking & $7(50)$ & $3(18.8)$ & $\mathrm{ns}$ \\
\hline Alcoholism & $3(21.4)$ & $2(12.5)$ & $\mathrm{ns}$ \\
\hline Comorbidities, n (\%) & $6(42.9)$ & $6(37.5)$ & $\mathrm{ns}$ \\
\hline Asthma & $1(7.1)$ & $0(0)$ & $\mathrm{ns}$ \\
\hline COPD & $2(14.3)$ & $3(18.8)$ & $\mathrm{ns}$ \\
\hline Diabetes Mellitus & $2(14.3)$ & $5(31.3)$ & $\mathrm{ns}$ \\
\hline Heart Failure & $0(0)$ & $1(6.3)$ & $\mathrm{ns}$ \\
\hline Chronic Kidney Disease & $2(14.3)$ & $0(0)$ & $\mathrm{ns}$ \\
\hline Hematologic Disease & $0(0)$ & $0(0)$ & $\mathrm{ns}$ \\
\hline Obesity & $4(28.6)$ & $2(12.5)$ & $\mathrm{ns}$ \\
\hline \multicolumn{4}{|l|}{ Previous Treatment, n (\%) } \\
\hline Corticosteroids & $0(0)$ & $0(0)$ & $\mathrm{ns}$ \\
\hline Statins & $4(28.6)$ & $4(25)$ & $\mathrm{ns}$ \\
\hline Insulin & $2(14.3)$ & $0(0)$ & $\mathrm{ns}$ \\
\hline \multicolumn{4}{|l|}{ ICU Treatment, n (\%) } \\
\hline Corticosteroids & $10(71.4)$ & $6(37.5)$ & $\mathrm{ns}$ \\
\hline NMB & $10(71.4)$ & $4(25)$ & 0.014 \\
\hline Days with NMB, mean (SD) & $1(1-2)$ & $1(0-2)$ & $\mathrm{ns}$ \\
\hline Insulin & $12(85.7)$ & $15(93.8)$ & $\mathrm{ns}$ \\
\hline Rehabilitation & $0(0)$ & $1(6.3)$ & $\mathrm{ns}$ \\
\hline Benzodiazepines & $7(50)$ & $14(87.5)$ & 0.032 \\
\hline Days with benzodiazepines, mean (SD) & $0.5(0-2)$ & $2.5(2-6.5)$ & 0.013 \\
\hline
\end{tabular}




\begin{tabular}{|c|c|c|c|}
\hline Opioids & $9(64.3)$ & $16(100)$ & 0.014 \\
\hline Days with opioids, mean (SD) & $1.5(0-3)$ & $4.5(2-6.75)$ & 0.007 \\
\hline Vasoactive Drugs & $13(92.9)$ & $12(75)$ & $\mathrm{ns}$ \\
\hline Maximum dose, $\mu \mathrm{g} / \mathrm{kg} / \mathrm{min}$, mean (SD) & $1.3(0.8)$ & $0.67(1.35)$ & $\mathrm{ns}$ \\
\hline Enteral Nutrition & $5(35.7)$ & $13(81.3)$ & 0.014 \\
\hline Days with enteral nutrition. mean (SD) & $0(0-2)$ & $7(3.35-11.5)$ & $<0.001$ \\
\hline Total Parenteral Nutrition & $0(0)$ & $0(0)$ & ns \\
\hline Infectious complications, $\mathrm{n}(\%)$ & $2(14.3)$ & $11(68.8)$ & 0.004 \\
\hline Non-infectious complications, $\mathrm{n}(\%)$ & $2(14.3)$ & $5(31.3)$ & ns \\
\hline
\end{tabular}

Table 2. Clinical characteristics of included subjects

\begin{tabular}{|c|c|c|c|c|}
\hline & $\begin{array}{c}\text { Control } \\
n=5\end{array}$ & $\begin{array}{c}\text { Brain-dead } \\
n=14\end{array}$ & $\begin{array}{l}\text { Maastricht III } \\
n=16\end{array}$ & $\mathbf{p}$ \\
\hline Diaphragm, $\%$, mean (SD) & & & & \\
\hline Total abnormal muscle fibers & $3.5(1.1)$ & $9.4(10.7)$ & $6.5(14.2)$ & 0.709 \\
\hline Internal nuclei & $2.4(1.0)$ & $0.3(0.3)^{* *}$ & $1.1(1.5)^{\Psi}$ & 0.003 \\
\hline Inflammatory cells & $0.5(0.3)$ & $0.5(0.1)$ & $0.5(0.1)$ & 0.392 \\
\hline Lipofuscin deposits & $0.4(0.1)$ & $7.9(10.5)^{* * *}$ & $4.2(12.2)$ & 0.000 \\
\hline Abnormal viable & $0.2(0.1)$ & $0.1(0.2)$ & $0.1(0.2)$ & 0.748 \\
\hline Inflamed/necrotic & $0(0)$ & $0.6(0.4)$ & $0.6(0.5)$ & 0.485 \\
\hline \multicolumn{5}{|l|}{ Quadriceps, \%, mean (SD) } \\
\hline Total abnormal muscle fibers & $2.4(0.6)$ & $2.3(5,0)$ & $1,8(2,4)$ & 0.730 \\
\hline Internal nuclei & $1.0(0.5)$ & $0.2(0,4)$ & $0,5(0,8)$ & 0.247 \\
\hline Inflammatory cells & $0.1(0.1)$ & $0(0)$ & $0.1(0.1)$ & 0.343 \\
\hline Lipofuscin deposits & $1.3(0.5)$ & $2.0(5.0)$ & $1.0(2.2)$ & 0.487 \\
\hline
\end{tabular}




\begin{tabular}{|l|c|c|c|c|}
\hline Abnormal viable & $0(0)$ & $0(0)$ & $0(0)$ & 0.223 \\
\hline Inflamed/necrotic & $0(0)$ & $0.1(0.4)$ & $0.2(0,3)$ & 0.787 \\
\hline
\end{tabular}

Table 3. Histological evaluation of muscle structure abnormalities in the three groups. ** Between Braindead and Control, $\mathrm{p}<0.01$; *** Between Brain-dead and Control, $\mathrm{p}<0.001 ; \Psi$ Between Maastricht III and Control, $\mathrm{p}<0.05$. See the categories and definitions for point counting in the Table 1, Online Supplement.

\title{
Online Supplement
}

\section{Structural differences in the diaphragm of patients following controlled vs assisted and spontaneous mechanical ventilation}

\author{
Marin-Corral J, Dot I, Boguña M, Cecchini L, Zapatero A, Gracia MP, Pascual-Guardia
} S, Vilà C, Castellví A, Pérez-Terán P, Gea J, Masclans JR

\section{Methods}

This original single-center prospective interventionist cohort study was conducted at the Critical Care Department of the Hospital del Mar in Barcelona and by the Critical illness research group (GREPAC) at the Hospital del Mar's Institute of Medical Investigations (IMIM). It was designed in accordance with our institution's ethical standards on human experimentation established in accordance with the 1964 Helsinki Declaration and its subsequent amendments. The study was approved by our Local Ethics and Clinical Research Committee (IRB PSMAR, 2017/7183/I) and by the Catalan Transplant Organization (OCATT). Written informed consent was obtained from all relatives prior to the study. Any details that might disclose the subject's identity in the study were omitted.

The sample size was based on previous data regarding organ donation and was calculated in order to find significant differences between two independent means in terms of muscle atrophy. Accepting an alpha risk of 0.05 and a beta risk of less than 0.2 in a bilateral contrast, 11 subjects in the MS group and 11 subjects in the BD group were required in order to detect a difference equal to or greater than 600 units $\left(\mu \mathrm{m}^{2}\right)$. It was assumed that the common standard deviation was 400 , and a follow-up loss rate of $5 \%$ was estimated.

\section{Subjects}

MS donors. Non heart-beating donors were donors diagnosed as dead due to irreversible cessation of cardiorespiratory functions (after cardiac arrest). MS donors included patients with non-survivable injuries in whom the treating clinicians decided to withdraw treatment and not commence resuscitation 
(limiting life support or LLS) after cardiac arrest, in the ICU or in the operating room. The predictability or control of cardiorespiratory arrest allowed procedures that avoided organic hypoperfusion by administering cold perfusions or the initiation of extracorporeal circulation after cardiac arrest, which kept the tissues in a suitable condition. The potential MS donors presented few important comorbidities, which predicted a good viability of the organs and they usually were able to generate spontaneous respiratory activity during MV. As a result, SMV ventilation was possible.

$B D$ organ donors. Donors with a $\mathrm{BD}$ diagnosis. One of criteria for $\mathrm{BD}$ diagnosis was the lack of spontaneous respiratory muscle activity during normocapnia as well as normoxic hypercapnia. These data reveal that $\mathrm{DF}$ of $\mathrm{BD}$ organ donors were completely inactive during the period of $\mathrm{MV}$ following the diagnosis, and in most cases previous to it.

CTL subjects. Patients without significant comorbidities who underwent thoracotomy of a suspected early localized lung malignancy and who needed several hours of MV. All subjects were on bronchodilators and inhaled corticosteroids.

\section{Biopsies}

MS donors. DF biopsies in MS donors were obtained in the operating room, after withdrawing life support treatment (MV) and certification of death. At that time, in order to avoid ischemia due to hypoperfusion, the extracorporeal membrane oxygenation (ECMO) or cold in situ perfusion were started. In a few cases where only kidneys were suitable for retrieval, a rapid extraction was performed without reperfusion measures. Specimens were taken after a variable period of MV. The QD biopsy was extracted in the ICU, under aseptic conditions and during the four hours before the organ donation surgery.

$B D$ organ donors. DF biopsies in BD organ donors were obtained in the operating room at the time of organ extraction, prior to circulatory arrest and as soon as was possible during organ removal, ensuring minimal ischemia time due to hypoperfusion. Specimens were taken after a variable period of MV. The QD biopsy was extracted in the ICU, under aseptic conditions and in the four-hour period prior to the organ donation surgery.

CTL subjects. DF biopsies in CTL subjects were obtained at the end of the surgery and before reversal of the neuromuscular blockade. In this case, DF of CTL patients was inactive for a short period of time prior to biopsy. QD biopsy was obtained at the same time as DF biopsy, at the end of the surgery, before reversal of the neuromuscular blockade.

\section{Immunohistochemical data}

Morphometric analyses were carried out in the DF and QD samples. Muscle samples were immersed in subsequent baths of different degrees of alcohol, formol, and xylol, to be finally embedded in paraffin. Three-micrometer muscle paraffin-embedded sections were obtained using a microtome and fixed on a glass slide which was then dried by heat $\left(60^{\circ} \mathrm{C}\right)$. All sections were deparaffinized and incubated with 
citric acid solution in a pressure cooker (antigen retrieval protocol). Slides were then incubated in a humid chamber with monoclonal anti-myosin heavy chain-I (clone MHC, Biogenesis Inc., Poole, UK) and monoclonal anti-myosin heavy chain-II antibodies (clone MY-32, Sigma-Aldrich, Saint Louis, MO, USA). After several washes in phosphate-buffered saline, slides were incubated with biotinoylated secondary antibodies followed by HRP-conjugated streptavidin and diaminobenzidine (LSAB + HRP kit, DakoCytomation Inc, CA, USA) as a substrate. Negative control slides were exposed only to secondary antibodies. Slides were counterstained with hematoxylin, dehydrated, and mounted for conventional microscopy. The cross-sectional area (CSA), mean least diameter, and proportions of type I (expressing MHCI) and type II (expressing MHCII) fibers were assessed by using a light microscope (Olympus, Series BX50F3, Olympus Optical Co., Hamburg, Germany) coupled with an image-digiting camera (Pixera Studio, version 1.0.4; Pixera Corporation, Los Gatos, CA) and evaluated using specific morphometry program (Image J, U. S. National Institutes of Health, Bethesda, Maryland, USA). At least 150 fibers were measured and counted in each muscle specimen.

\section{Muscle structure abnormalities}

The area fraction of normal and abnormal muscle (abnormal cellular events taking place in the muscle fibers) was evaluated on $3 \mu \mathrm{m}$ paraffin-embedded sections of DF and QD fixed on a glass slide. They were stained with hematoxylin and eosin. Following the method of point counting used by Macgowan et $a l$ and other authors [29, 30], each image of muscle cross section was quantified by projecting 63 pointintercepts on each image sample. Two independent observers classified each point-intercept in one of the following categories: normal structure muscle, presence of internal nucleus, inflammatory cells, lipofuscin deposits, abnormal viable fibers or inflamed or necrotic fibers (Table 1 Online Supplement). At a magnification of $\mathrm{x} 40$, each point-intercept was assigned to a specific category. The fraction areas of normal and abnormal muscle were determined by calculating the proportion of points in each assigned category. The sum of the percentages of the non-normal categories was called total abnormal muscle fibers. Photographs were used for reference when assigning point-intercepts to categories. For each muscle cross section, all possible non-overlapping fields were counted in a standardized manner. The observer was blinded to the identity of the slide.

\section{Discussion}

In this study, there were no differences in the atrophy or in the fiber percentages presented in any ventilated donor group between the two fiber types; however, when we compared MSIII donors according to the type of preservation, we found that those who had been administered cold perfusion spent more hours in CMV and without diaphragm stimuli than rapid extraction although they spent the same number hours under MV. In accordance with previous data, only MHCII CSA were significantly decreased compared with rapid extraction, and there were no differences in the other clinical characteristics. In this regard, both short-term and long-term CMV result in modifications of the MHC phenotype in rats. Within 12 and $18 \mathrm{~h}$ of CMV both type I and II fibers are reduced in size, but type II fibers exhibit a much greater reduction; within $24 \mathrm{~h}$ of MV the transcript levels of the MHCII isoforms fall by $20 \%$, consistent with 
the preferential atrophy observed in the above-mentioned studies. This modification of the MHC phenotype could contribute to the force decline of the diaphragm reported in some articles, since slow fibers produce less force than fast fibers [37].

\begin{tabular}{lllll}
\hline A & Time on MV & Total time on MV & $\begin{array}{l}\text { With and without diaphragm } \\
\text { stimuli }\end{array}$ \\
\hline B & Time on CMV & Time on controlled modalities & $\begin{array}{l}\text { With and without diaphragm } \\
\text { stimuli }\end{array}$ \\
B1 & CMV with RRR = PRR & $\begin{array}{l}\text { Time on CMV with ventilations } \\
\text { totally controlled by the ventilator }\end{array}$ & Without diaphragm stimuli \\
B2 & CMV with RRR $>$ PRR & $\begin{array}{l}\text { Time on CMV with ventilations } \\
\text { assisted by the ventilator }\end{array}$ & With diaphragm stimuli \\
\hline C & Time on SMV & Time on spontaneous modalities & With diaphragm stimuli \\
& &
\end{tabular}

B1 $=$ Time without diaphragm stimuli

$\mathrm{B} 2+\mathrm{C}=$ Time with diaphragm stimuli

MV: mechanical ventilation; CMV: controlled mechanical ventilation; RRR: real respiratory rate; PRR: predetermined respiratory rate; SMV: spontaneous mechanical ventilation. 
Table 1. Definitions for the ventilatory variables. 


\begin{tabular}{lll}
\hline Category & Name & Definition \\
\hline 1 & Normal muscle & $\begin{array}{l}\text { Polygonal fiber, acidophilic cytoplasm, plasma membrane, } \\
\text { peripheral muscle nuclei }\end{array}$ \\
2 & Internal nuclei & Fiber with at least one muscle nucleus located internally \\
3 & Inflammatory cell & Round-shaped nucleus in interstitial space \\
4 & Lipofuscin & Fiber containing deposits of yellow-brown pigmentation at least size \\
& Abnormal viable & Small fiber with two or more oblique angles or with basophilic \\
5 & Inflamed/necrotic & Fiber containing inflammatory cell(s); necrotic mass of \\
& & inflammatory cells and muscle debris
\end{tabular}

*Total count $=\Sigma$ Categories 1 to 6 ; area fraction normal muscle $=\Sigma$ Category $1 /$ Total count X 100; total abnormal muscle fibers $=\Sigma$ Category 2 to 6/Total count X 100. Modified from Macgowan et al, Am J Respir Crit Care Med 2001.

Table 2. Categories and definitions for point counting. 


\begin{tabular}{lccc}
\hline & $\begin{array}{c}\text { Control } \\
\mathbf{n = 5}\end{array}$ & $\begin{array}{c}\text { Brain-dead } \\
\mathbf{n = 1 4}\end{array}$ & $\begin{array}{c}\text { Maastricht III } \\
\mathbf{n = 1 6}\end{array}$ \\
\hline & & & \\
Diaphragm, \%, mean (SD) & & & $51.83(9.09)$ \\
$\quad$ Type I fiber & $52.60(3.25)$ & $49.07(10.25)$ & $48.17(9.09)$ \\
$\quad$ Type II fiber & $47.40(3.25)$ & $50.93(10.25)$ & \\
Quadriceps, \%, mean (SD) & & & $45.36(6.71)$ \\
Type I fiber & $41.66(14.10)$ & $39.86(10.16)$ & $54.63(6.71)$ \\
Type II fiber & $58.33(14.10)$ & $60.14(10.17)$ & \\
\hline
\end{tabular}

Table 3. Proportion of type I and II muscle fibers in the three cohort groups.

\begin{tabular}{lccc}
\hline & $\begin{array}{c}\text { Cold perfusion } \\
\mathbf{n = 5}\end{array}$ & Rapid extraction & $\mathbf{p}$ \\
& $196(143-386)$ & $208(141-348)$ & $\mathrm{ns}$ \\
\hline Total MV, h, median (IQR) & $177(122-197.5)$ & $75(40-154)$ & 0.022 \\
Total CMV, h, median (IQR) & $159(122-187)$ & $58(30-110)$ & 0.004 \\
Diaphragm without stimuli, h, median (IQR) & & & \\
Diaphragm, $\mu \mathrm{m}^{2}$, mean $(\mathrm{SD})$ & $1145.6(383.4)$ & $1631.1(789.7)$ & $\mathrm{ns}$ \\
$\quad$ Type I fiber & $933.5(442.8)$ & $1869.4(1108.7)$ & 0.047 \\
$\quad$ Type II fiber & & & \\
\hline
\end{tabular}

Table 4. Characteristics of MS donors in two types of organ preservation, cold perfusion or rapid extraction. 


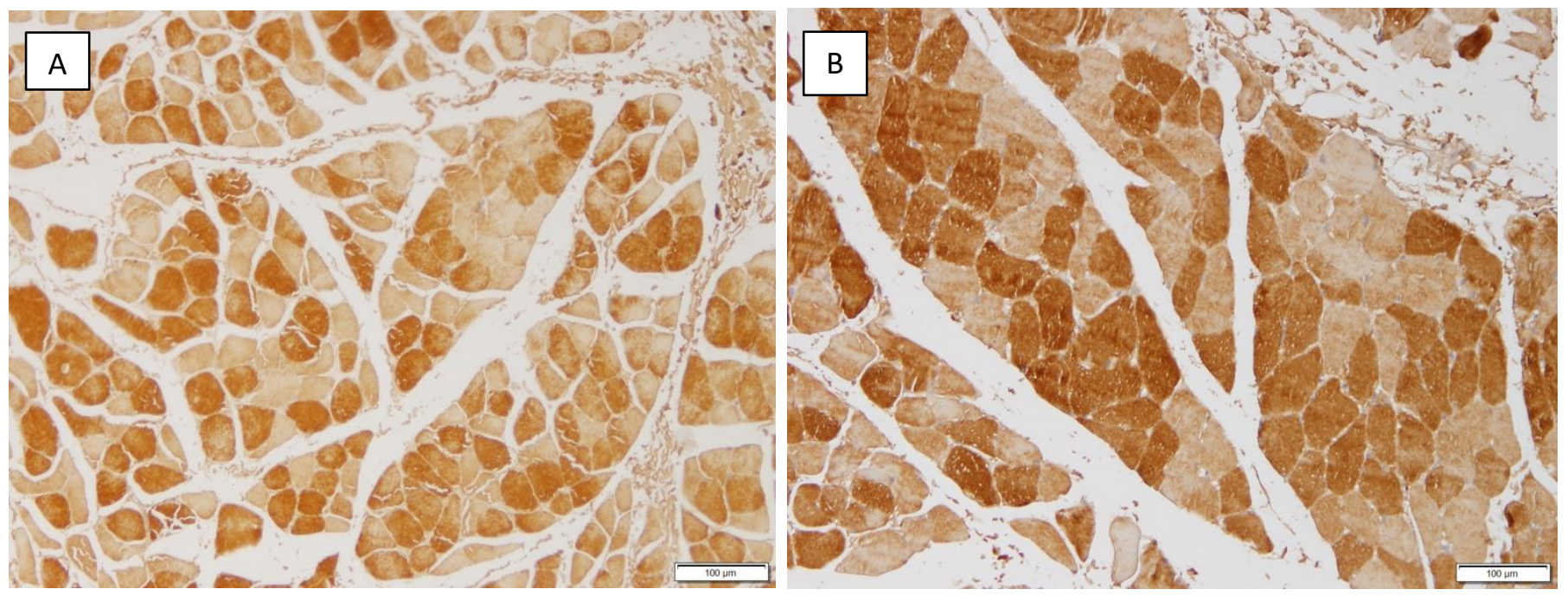

Figure 1. Comparison of representative MSIII donor diaphragm-biopsy specimens with respect to fiber 
size and MHCI/MHCII proportions. A: MHCI immunohistochemical staining of a MSIII donor who spent 100 or more hours without diaphragm stimuli (10X). B: MHCI immunohistochemical staining of a MSIII donor who spent less than 100 hours without diaphragm stimuli (10X).
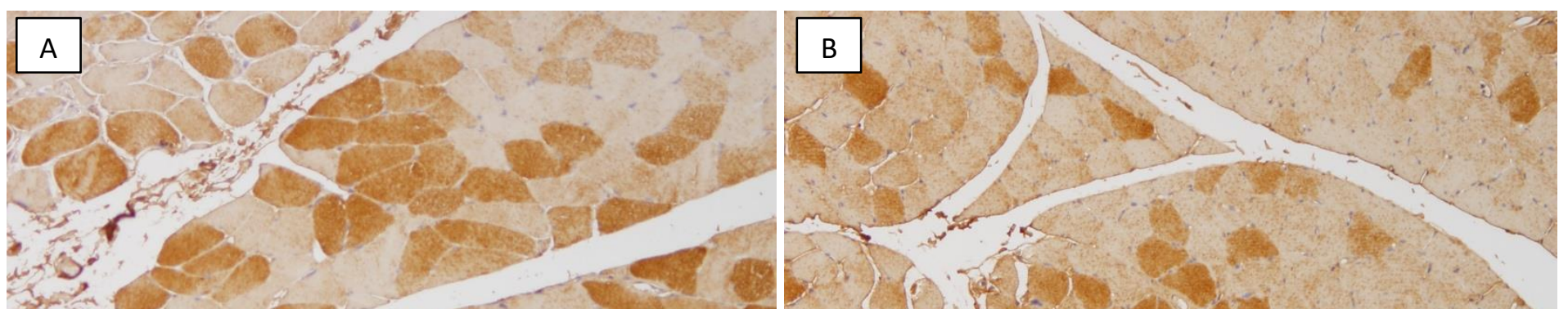
Figure 2. Comparison of representative BD and MSIII donor quadriceps-biopsy specimens with respect to fiber size and MHCI/MHCII proportions. A: MHCI immunohistochemical staining of a BD donor (10X). B: MHCI immunohistochemical staining of a MSIII donor (10X). 

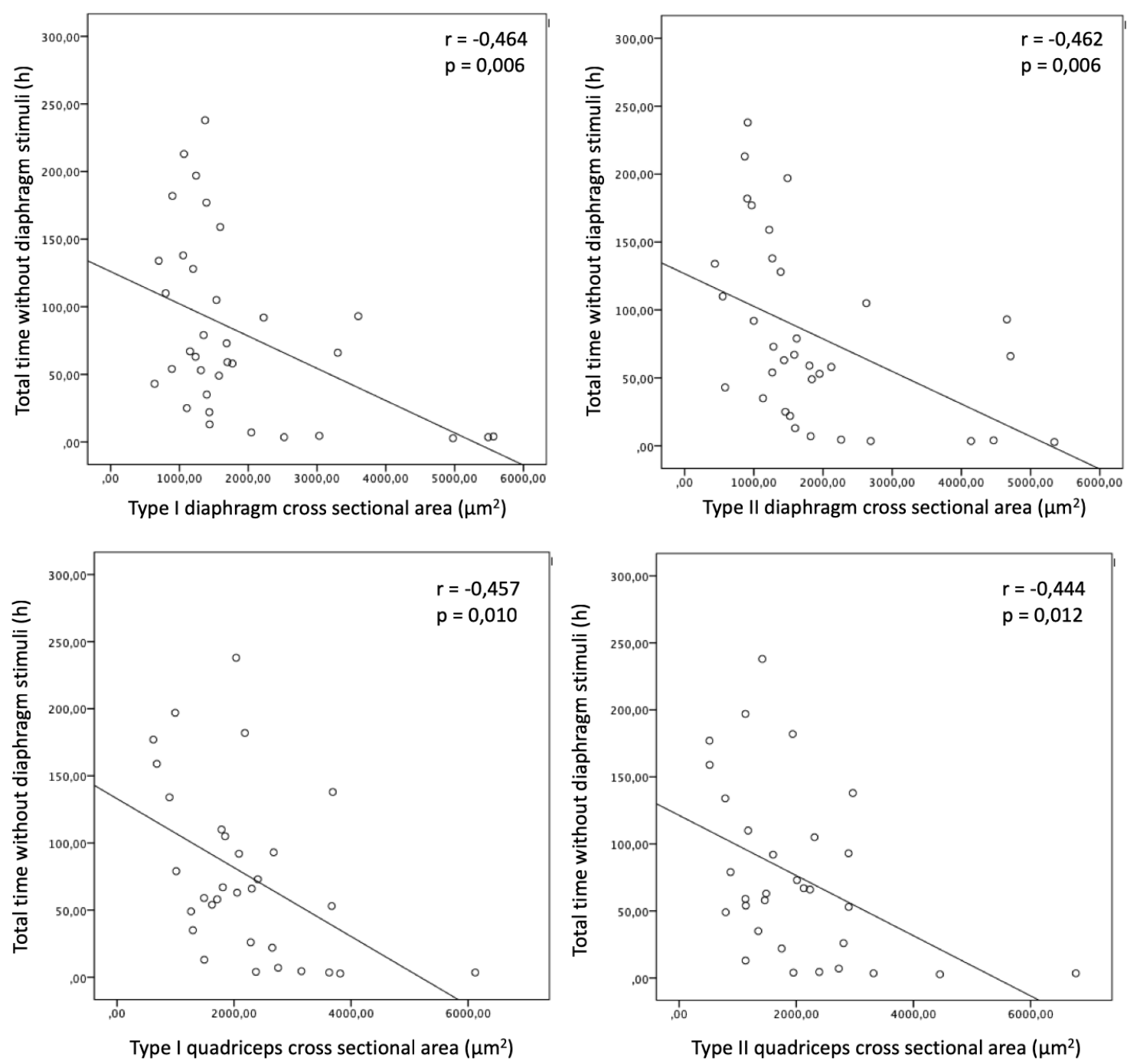

Figure 3. Correlations between type I and type II diaphragm and quadriceps cross sectional area and time spent without diaphragm stimulation. 

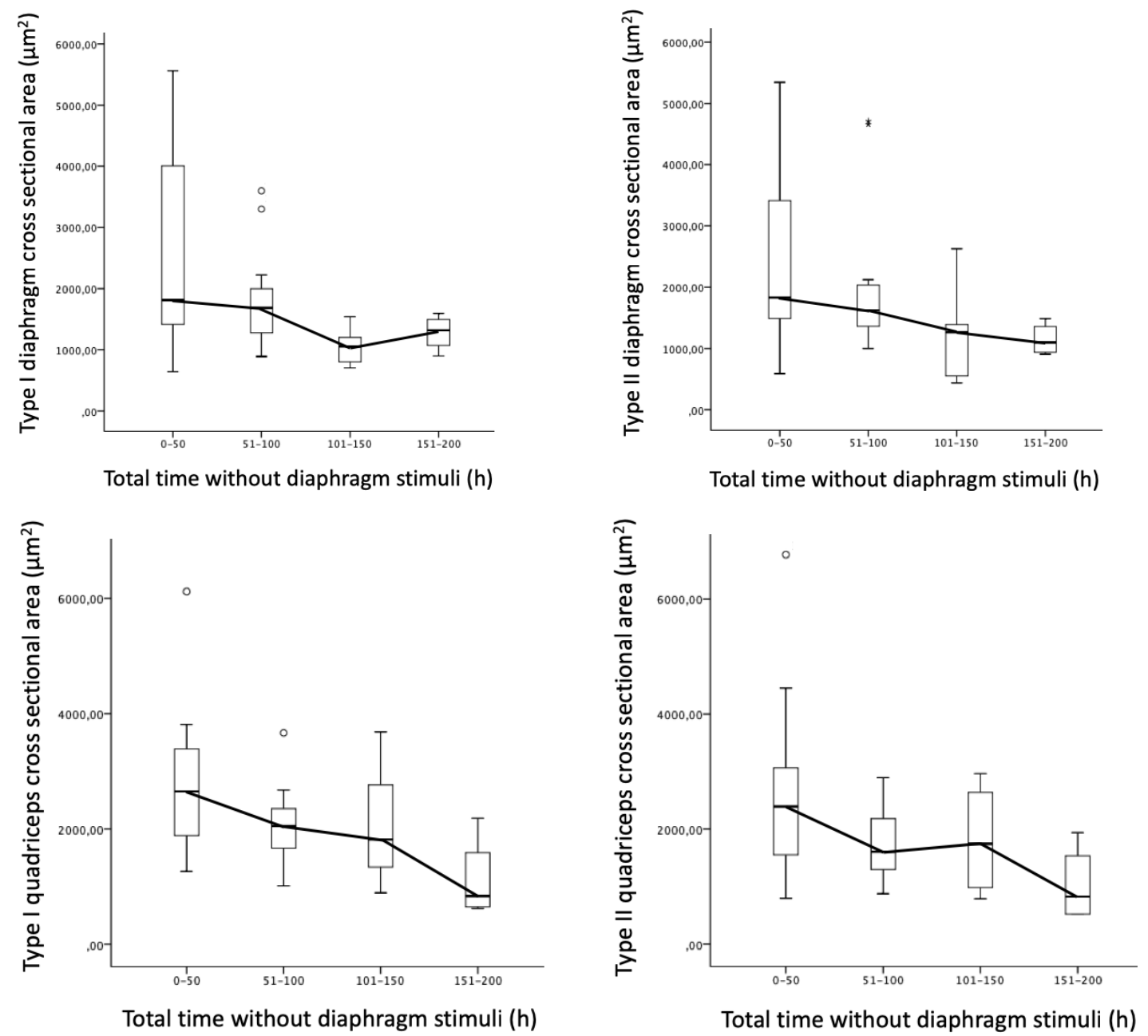

Figure 4. Diaphragm and quadriceps cross sectional area according to total time in controlled mechanical ventilation without diaphragm stimuli. 

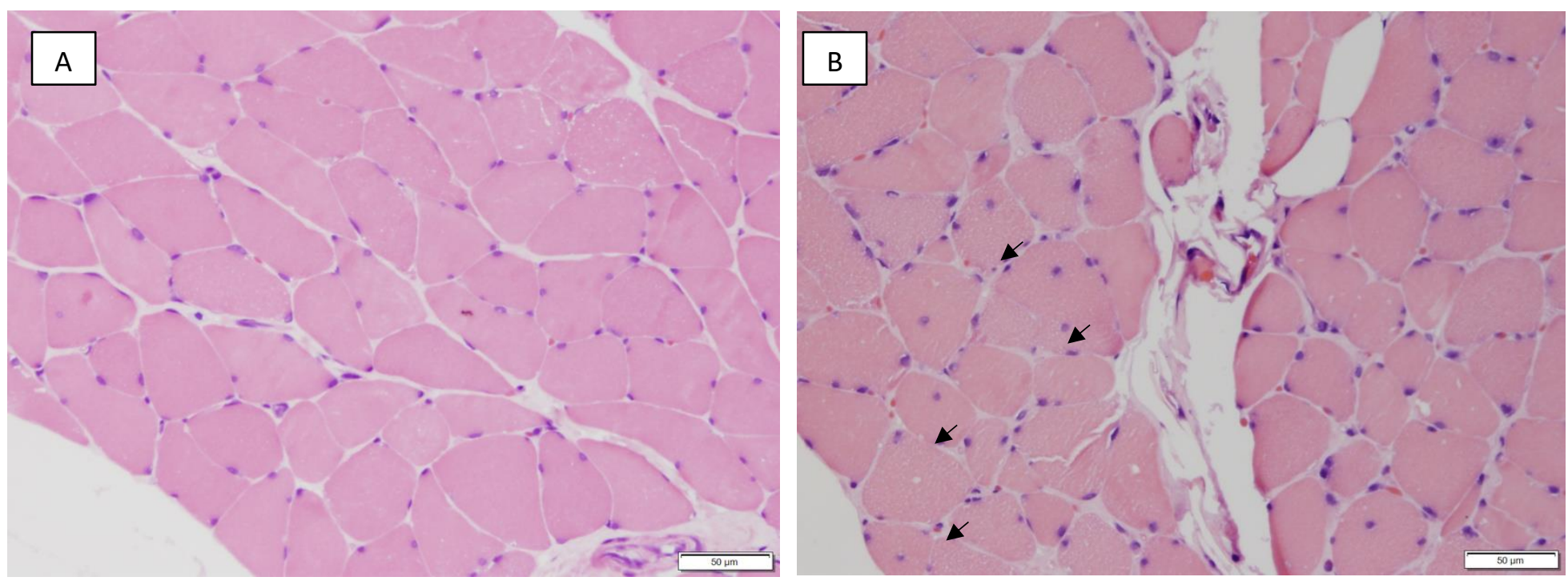

Figure 5. Comparison of representative BD and MSIII donor diaphragm-biopsy specimens with respect to muscle abnormalities (black arrows indicate internal nuclei) A: Hematoxylin-eosin staining of a BD donor (20X). B: Hematoxylin-eosin staining of a MSIII donor (20X). 

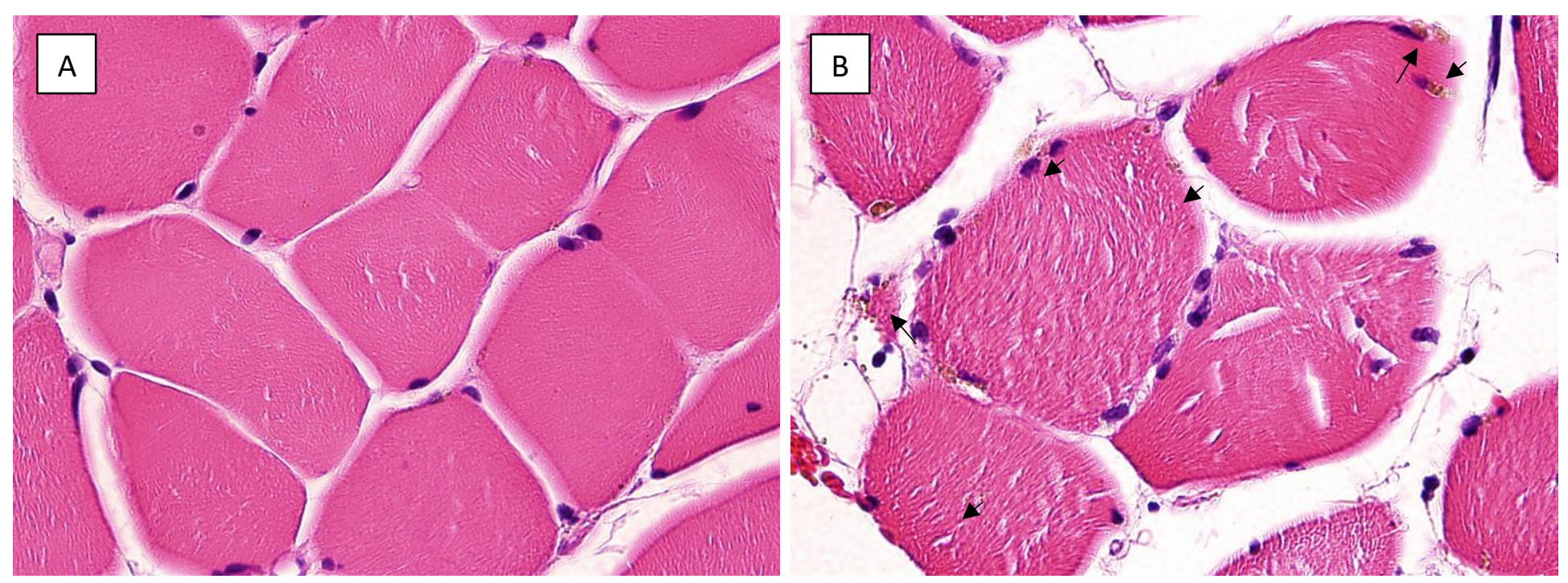
Figure 6. Comparison of representative CTL and BD donor diaphragm-biopsy specimens with respect to muscle abnormalities (black arrows indicate lipofuscin deposits) A: Hematoxylin-eosin staining of a CTL subject (X40). B: Hematoxylin-eosin staining of a BD donor (X40). 
